\title{
Intelligent Ping Sequencing for Multistatic Sonar Systems
}

\author{
David W. Krout and Mohamed A. El-Sharkawi \\ Department of Electrical Engineering \\ University of Washington \\ Campus Box 352500 \\ Seattle, WA 98195-2500 \\ \{dkrout,elsharkawi\}@ee.washington.edu
}

\author{
Warren L. J. Fox and Megan U. Hazen \\ Applied Physics Laboratory \\ University of Washington \\ 1013 NE 40th St. \\ Seattle, WA 98105-6698 \\ \{warren,megan\}@apl.washington.edu
}

\begin{abstract}
We study the problem of determining optimal pinging strategies in multistatic sonar systems with multiple sources. We are specifically investigating algorithms that determine optimal pinging strategies both for generalized search scenarios, and for holding confirmed target tracks with constraints related to maintaining search performance in the rest of the area. An important part of this work is the development of metrics to be used in the optimization procedures. For maintaining search coverage, we used a "probability of target presence" metric formulation. This formulation utilizes sonar performance prediction and a Bayesian update equation to incorporate negative information (i.e., searching an area but finding no targets). We also discuss strategies that can be used to increase the performance of a multistatic field, such as the use of bandwidth diversity.
\end{abstract}

Keywords: Tracking, data association, sensor management, distributed sensors, resource allocation.

\section{Introduction}

Distributed sensor fields are used to track targets in large areas. There are many practical and operational issues associated with the operation of these fields such as synchronization, optimal tracking architecture, and sensor management [1]. This paper is predominantly concerned with sensor management, where the goal is typically defined as optimizing the performance of area search and tracking by the allocation of sensor resources. In other words, sensor management attempts to maximize system performance by optimally utilizing the emplaced sensors. A few overall approaches to sensor management are presented in $[2,3,4]$, which mainly focus on the degrees of freedom of the sensors. One example is the electronically scanned antenna (ESA) system, which has an adjustable aperture that can be quickly redirected to improve tracking performance.

We are specifically interested in distributed active sonar systems, i.e., networks of multiple sonobuoys consisting of sonar sources and receivers. The sources transmit acoustic energy into the water, and the receivers listen for echoes reflected from targets.
Sonobuoy fields can be used for several different missions such as area search, or track and hold. When targets are detected, it is important to be able to track them, but at the same time to continue monitoring the rest of the area for other potential targets entering the search space.

This paper explores the sensor management of multistatic active sonar systems, where we control the ping sequence of multiple transmitters in a distributed sensor field. In the next section, we will discuss the framework for the problem followed by a formulation of a metric, which will be used in the search aspect of intelligent ping sequencing. In Section 3 we will discuss ping management strategies when targets are detected using track quality metrics and bandwidth diversity.

\section{Distributed Search}

We assume that we start with a near optimum placement of transmitters and receivers [5]. For a large field of sensors it is difficult to know which sensor is the best choice to ping. In the past, systems have used simple approaches like pinging in sequential order or random selection. These approaches can work when the number of transmitters is small, but in larger and more complex systems, a more intelligent method needs to be devised to manage the ping sequence. We will show examples when targets are not detected without intelligent ping sequencing later in the paper. If targets are detected, the ping sequence must allow for holding track while maintaining search performance in the rest of the area. It is necessary to develop metrics to drive these optimizations. This section describes the metrics and "bookkeeping" procedures we developed for optimizing the search procedures using a multistatic sonar system.

\subsection{Probability of Target Presence}

Here we define a metric called "Probability of Target Presence," $P_{T}(x, y)$. This should be interpreted as the probability that a target is present at geographic coordinates $(x, y)$. At this stage of our research we are not considering the depth of the water column in our analysis, so $P_{T}$ is a function of only the lateral coordinates 
$x$ and $y$. Extension to include the depth dimension is in progress. $P_{T}$ equal to one means that we know a target exists, and a $P_{T}$ of zero indicates that we know a target does not exist at the location. $P_{T}$ equal to 0.5 would then correspond to an unknown state. For the problem of area search, we discretize the search area into cells of equal size and assign an a priori probability of target presence. We assume no detections are made and we remain in search mode. Using Bayes theorem we can define the probability of target presence given no detections as

$$
P_{T \mid N D}=\frac{P_{N D \mid T} P_{T}}{P_{N D \mid T} P_{T}+P_{N D \mid N T} P_{N T}},
$$

where $N D$ denotes no detection, $N T$ denotes no target present, and $T$ denotes target presence. Table 1 shows how these different quantities are calculated based on the definition of $P_{d}$ and $P_{f a}$.

Table 1: Decision Matrix

\begin{tabular}{|c|c|c|c|}
\hline \multicolumn{2}{|c|}{} & \multicolumn{2}{c|}{ Decision } \\
\cline { 3 - 4 } \multicolumn{2}{|c|}{} & $\begin{array}{c}\text { Target } \\
\text { Present }\end{array}$ & $\begin{array}{c}\text { No Target } \\
\text { Present }\end{array}$ \\
\hline \multirow{3}{*}{ At Input } & $\begin{array}{c}\text { Target } \\
\text { Present }\end{array}$ & $\mathrm{Pd}$ & $1-\mathrm{Pd}$ \\
\cline { 2 - 4 } & $\begin{array}{c}\text { No Target } \\
\text { Present }\end{array}$ & $\mathrm{Pfa}$ & $1-\mathrm{Pfa}$ \\
\hline
\end{tabular}

$P_{d}$ is the probability that we detect a target when a target actually is present, and $P_{f a}$ is the probability that we decide a target is present when there is no actual target (false alarm). Sonar performance predictions are typically generated in terms of signal excess (SE) via manipulation of the sonar equation[6]. Signal excess is defined as the signal-to-noise ratio (SNR) minus a detection threshold (DT), where DT is set based on design values of $P_{d}$ (usually set to 0.5 ) and $P_{f a}$ (usually set to a very low number, like $10^{-5}$ ). Signal excess equal to $0 \mathrm{~dB}$ indicates the design $P_{d}$, and analytic representations are available to calculate $P_{d}$ for other values of SE. See [7] for further discussion on this topic.

Figure 1 is an example of a SE map, assuming a homogeneous environment and uniform target strength vs. aspect. The squares and diamonds indicate locations of receivers and sources, respectively. The figure represents the signal excess for one source pinging (the large circle), and is calculated as the maximum signal excess that would be seen at any receiver for a hypothetical target at that location. The light area represents a signal excess less than zero and the dark area represents signal excess greater than zero.

Substituting the values from Table (1) into Equation (1) gives the following equation:

$$
P_{T \mid N D}=\frac{\left(1-P_{d}\right) P_{T}}{\left(1-P_{d}\right) P_{T}+\left(1-P_{f a}\right)\left(1-P_{T}\right)} .
$$

The a posteriori $P_{T}$ for the given ping becomes the $a$ priori $P_{T}$ for the next ping. This approach allows us to

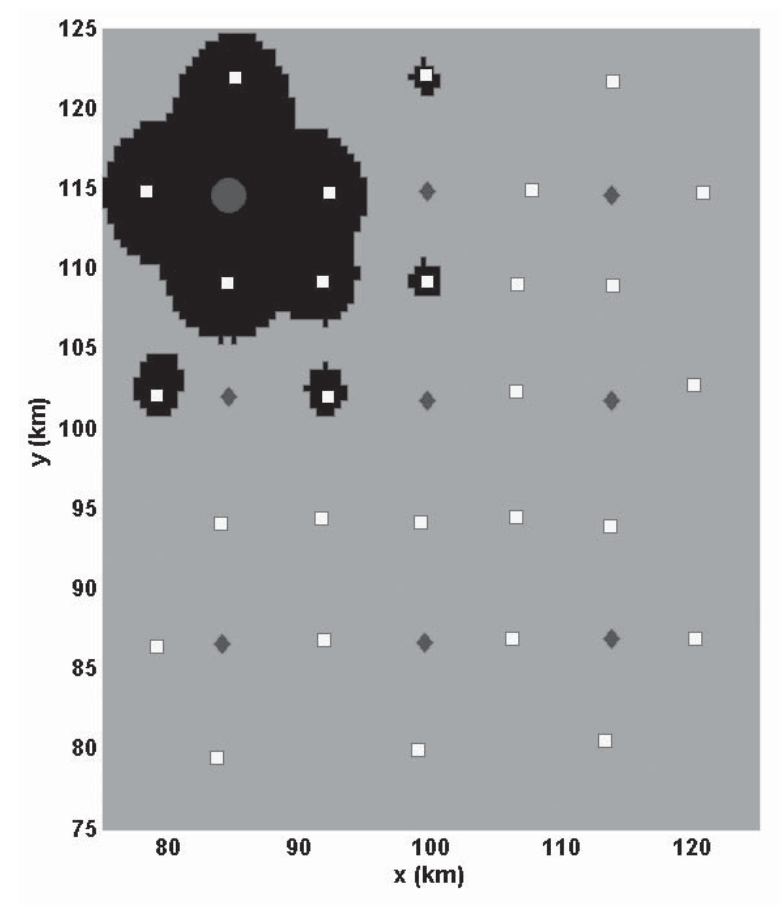

Figure 1: The signal excess map for one source pinging in a homogeneous environment. The large circle in the upper left is the source pinging. The dark area represents a signal excess greater than zero $\mathrm{dB}$.

incorporate negative information into a "bookkeeping" procedure that keeps track of where our search effort has been expended, and provides a metric that can be used in optimization routines as to where future search efforts can best be used.

This formulation does not account for targets moving into an area that has already been searched. Kanchanavally proposed a method for calculating the uncertainty in a target's movement based on Brownian motion [8]. We expand on this approach and apply it to the Probability of Target Presence in the next section.

\subsection{Accounting for Moving Targets}

The above formulation assumes stationary targets, however, we would also like to take into account the fact that targets may move into a previously searched area. Similar to the approach used in [8], we model a drift and diffusion process using the Fokker-Planck equation and Brownian motion. We first look at the one dimensional case where we model the movement of possible targets as a Wiener Process with and drift factor,

$$
d x \sim \sqrt{d t} N\left(m_{x} \cdot d t, 1\right)
$$

where $d t$ is the time increment, $d x$ is the stochastic process and $N$ is a normal distribution with a mean of $m_{x} \cdot d t$. What we would like to know is how the probability of target presence changes over time $(\Delta t)$ as a result of a random movement of the targets. In terms of a probability density function $\rho(x, t)$, the change 
over time is

$$
\rho(x, t+\Delta t)=\int_{-\infty}^{\infty} \rho(x-\Delta x, t) P(\Delta x, \Delta t) d \Delta x,
$$

where $P(\Delta x, \Delta t)$ is the probability of a target moving a distance $\Delta x$ in time $\Delta t$. Using a Taylor series expansion we obtain

$$
\begin{aligned}
& \rho(x, t+\Delta t) \cong \\
& \quad \int_{-\infty}^{\infty}\left[\rho(x, t)-\Delta x \frac{\partial \rho}{\partial x}+\frac{1}{2} \Delta x^{2} \frac{\partial^{2} \rho}{\partial x^{2}}\right] P(\Delta x, \Delta t) d \Delta x .(5)
\end{aligned}
$$

Defining the moments for $P$ from equation (3) we obtain the following:

$$
\begin{gathered}
\int_{-\infty}^{\infty} P(\Delta x, \Delta t) d \Delta x=1, \\
\int_{-\infty}^{\infty} \Delta x P(\Delta x, \Delta t) d \Delta x=m_{x} \Delta t, \text { and } \\
\int_{-\infty}^{\infty} \Delta x^{2} P(\Delta x, \Delta t) d \Delta x=\sigma^{2} \Delta t+m_{x}^{2} \Delta t^{2} .
\end{gathered}
$$

Combining the above results we obtain:

$$
\begin{aligned}
& \rho(\mathrm{x}, \mathrm{t}+\Delta \mathrm{t})-\rho(\mathrm{x}, \mathrm{t})= \\
& \quad \frac{-\partial \rho}{\partial x} m_{x} \Delta \mathrm{t}+\frac{1}{2} \frac{\partial^{2} \rho}{\partial x^{2}}\left(\sigma^{2} \Delta \mathrm{t}+m_{x} \Delta \mathrm{t}^{2}\right),
\end{aligned}
$$

and letting $\Delta t$ be small we obtain:

$$
\frac{\partial \rho}{\partial t}=-m_{x} \frac{\partial \rho}{\partial x}+\frac{1}{2} \sigma^{2} \frac{\partial^{2} \rho}{\partial x^{2}}
$$

Equation (10) is the Fokker-Planck equation in one dimension with a drift coefficient of $-m_{x}$ and a diffusion coefficient of $\frac{1}{2} \sigma^{2}$. Assuming we now have a two dimensional drift and diffusion process defined by

$$
\begin{gathered}
d x \sim \sqrt{d t} N\left(m_{x} \cdot d t, 1\right), \text { and } \\
d y \sim \sqrt{d t} N\left(m_{y} \cdot d t, 1\right),
\end{gathered}
$$

we can extend equation 10 to two dimensions obtaining

$$
\frac{\partial \rho}{\partial t}=-m_{x} \frac{\partial \rho}{\partial x}-m_{y} \frac{\partial \rho}{\partial y}+\frac{1}{2} \sigma_{x}^{2} \frac{\partial^{2} \rho}{\partial x^{2}}+\frac{1}{2} \sigma_{y}^{2} \frac{\partial^{2} \rho}{\partial y^{2}} .
$$

Comparing equation 13 to the standard form of the Fokker-Plank equation:

$$
\begin{array}{r}
\frac{\partial W}{\partial t}=\left[-\sum_{i=1}^{N} \frac{\partial}{\partial x_{i}} D_{i}^{(0)}(\{x\})+\right. \\
\left.\sum_{i, j=1}^{N} \frac{\partial^{2}}{\partial x_{i} \partial x_{j}} D_{i, j}^{(1)}(\{x\})\right] W
\end{array}
$$

we get the following coefficients for drift and diffusion:

$$
D_{i}^{(0)}=-m_{i}, \text { and }
$$

$$
D_{i}^{(1)}=\frac{1}{2} \sigma_{i}^{2} .
$$

We can then solve the Fokker-Planck equation by using finite difference methods. We first discretize the variables using the following:

$$
\begin{array}{cc}
x=i \Delta x_{g} & i=0,1,2, \ldots, n_{x} \\
y=j \Delta y_{g} & j=0,1,2, \ldots, n_{y} \\
t=k \Delta t & k=0,1,2, \ldots, n_{t},
\end{array}
$$

and apply finite difference methods to (13) obtaining

$$
\begin{aligned}
& \frac{\rho_{i, j, k+1}-\rho_{i, j, k}}{\Delta t}= \\
& \quad \frac{-m_{x}}{\Delta x_{g}}\left(\rho_{i, j, k}-\rho_{i-1, j, k}\right)+ \\
& \quad \frac{-m_{y}}{\Delta y_{g}}\left(\rho_{i, j, k}-\rho_{i, j-1, k}\right)+ \\
& \quad \frac{\sigma_{x}^{2}}{2\left(\Delta x_{g}\right)^{2}}\left(\rho_{i-1, j, k}-2 \rho_{i, j, k}+\rho_{i+1, j, k}\right)+ \\
& \frac{\sigma_{y}^{2}}{2\left(\Delta y_{g}\right)^{2}}\left(\rho_{i, j-1, k}-2 \rho_{i, j, k}+\rho_{i, j+1, k}\right) .
\end{aligned}
$$

Equation (20) is then solved for $\rho_{i, j, k+1}$ :

$$
\begin{aligned}
& \rho_{i, j, k+1}=\rho_{i, j, k}+\Delta t\left[\frac{-m_{x}}{\Delta x_{g}}\left(\rho_{i, j, k}-\rho_{i-1, j, k}\right)+\right. \\
& \frac{-m_{y}}{\Delta y_{g}}\left(\rho_{i, j, k}-\rho_{i, j-1, k}\right)+ \\
& \frac{\sigma_{x}^{2}}{2\left(\Delta x_{g}\right)^{2}}\left(\rho_{i-1, j, k}-2 \rho_{i, j, k}+\rho_{i+1, j, k}\right)+ \\
& \left.\frac{\sigma_{y}^{2}}{2\left(\Delta y_{g}\right)^{2}}\left(\rho_{i, j-1, k}-2 \rho_{i, j, k}+\rho_{i, j+1, k}\right)\right] \cdot
\end{aligned}
$$

Given we have the a priori distribution of $\rho$, we can then calculate the a posteriori distribution using the above equation.

Having defined $P_{T}$ as the probability of target presence in a cell, we are seeking to find how this probability changes over time due to target movement and the fact that we do not monitor each cell's status continuously. Equation (21) updates the movement of target probabilities. We can then apply this probability update Equation $(21)$ to $P_{T}$. We first initialize all the equally spaced cells to have a $P_{T}=.5$ which, as noted, earlier is a state of no information. When a sensor pings, we update the $P_{T}$ map using Eq. (2) and in the time before the next ping we update the $P_{T}$ map using Eq. (21). Assuming $\Delta t=1$, Equation (21) takes the form of a spatial filter whose impulse response looks like

\begin{tabular}{|c|c|c|}
\hline 0 & $\frac{\sigma_{y}^{2}}{2 \Delta y^{2}}$ & 0 \\
\hline$\frac{m_{x}}{\Delta x}+\frac{\sigma_{x}^{2}}{2 \Delta x^{2}}$ & $1-\frac{\sigma_{x}^{2}}{\Delta x^{2}}-\frac{\sigma_{y}^{2}}{\Delta y^{2}}-\frac{m_{x}}{\Delta x}-\frac{m_{y}}{\Delta y}$ & $\frac{\sigma_{x}^{2}}{2 \Delta x^{2}}$ \\
\hline 0 & $\frac{m_{y}}{\Delta y}+\frac{\sigma_{y}^{2}}{2 \Delta y^{2}}$ & 0 \\
\hline
\end{tabular}

To perform optimum search we can choose a ping sequence that minimizes the probability of target presence. For the current $P_{T}$ map we predict ahead one 


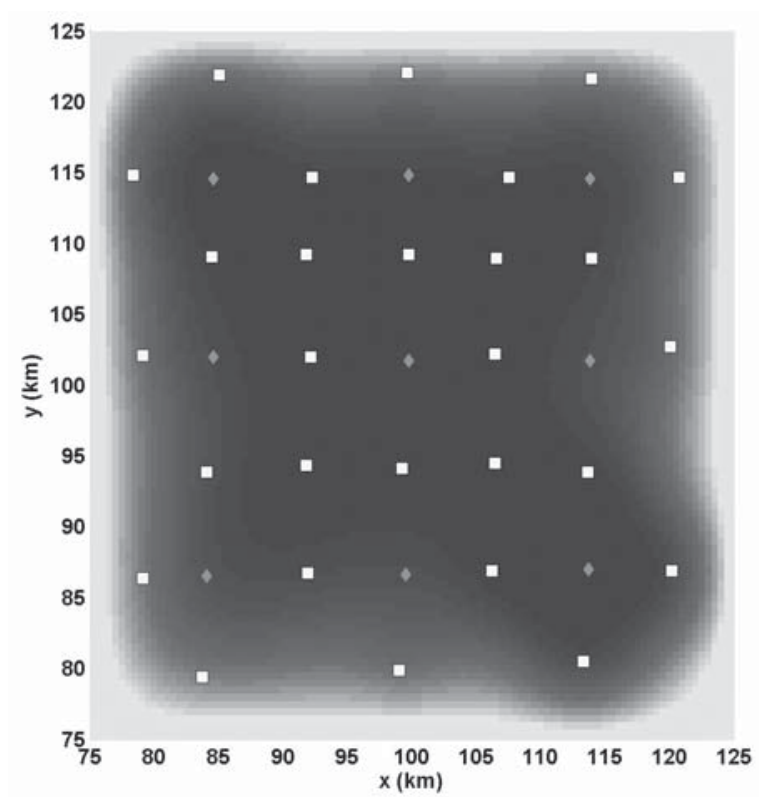

Figure 2: The probability of target presence for a diffusion only case. The dark area represents a $P_{T}$ of approximately zero

ping using the Bayes update equation (Eq. 2) for each possible choice of transmitter. The spatial filter is applied to account for targets that may have moved into the search space. We then choose the transmitter to ping that minimizes the sum of $P_{T}$ over the entire area. An example of this is shown in Fig. 2, which is a plot showing the probability of target presence after several pings. In this example targets are modeled with diffusion only. The squares and diamonds correspond to receivers and transmitters respectively. The darker area in the center represents a $P_{T}$ of approximately zero, and the edges where it is lighter represents a $P_{T}$ of approximately one half. The gradient from light to dark represents the probability of target presence increasing due to the chance that targets may move into an area already searched, which is calculated as described above. As $P_{T}$ increases near the edges (as seen in Fig. 2), intelligent ping sequencing selects transmitters close to the border to minimize the $P_{T}$.

\section{Track Management}

In the previous section we discussed a metric used in the area search mission when targets have not been detected. We will now discuss ping sequencing when a target or multiple targets have been detected. A simple approach for a ping sequence would be to continue pinging on the transmitter that gives the highest signal excess for the predicted target location. However, if there are multiple targets and false contacts it becomes important to have an intelligent ping sequence. For example, Fig. 3 shows a typical scenario with a false alarm rate of thirty, which means that there are thirty false contacts per ping. Contacts generated from targets and false alarms are represented by the large

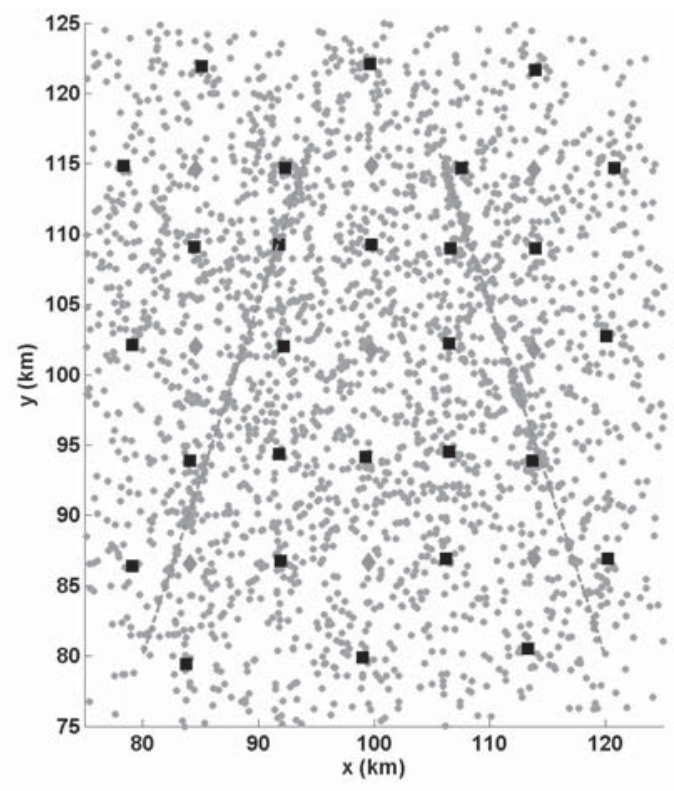

Figure 3: A plot displaying all the contacts, target originated and false contacts, for a random ping sequence case. This plot illustrates the number of false contacts that are generated during a possible tracking scenario.

dots. The figure shows all the contacts for the duration of the simulation. There are two real tracks for this scenario which are represented by dotted lines. The two targets move from the lower left and right corners to the top-middle of the search area. We will show in the following sections the improvement in tracking that ping sequencing can provide in these types of scenarios.

\subsection{Multiple Target Tracker}

In order to test ideas for intelligent ping sequencing we developed a multiple target tracker. Multiple target tracking (MTT) is a complex and difficult problem which has been studied quite extensively since the 1950's. We chose approaches to the MTT problem based on ease of implementation and proven robustness. Our tracker uses a modified version of nearest neighbor data association combined with a trackorientated multiple target tracking approach [4]. The tracks are processed with a basic Kalman Filter using a nearly constant velocity model. Logic based track maintenance is used to initiate and eliminate tracks $[4,9]$. We merge tracks based on the position and velocity estimates of the tracks.

\subsection{Track Quality}

We have investigated two different techniques for handling ping sequencing when there are multiple confirmed tracks. One technique is bandwidth diversity, discussed in the next section. In this section we look at track quality to determine which sources to ping. We define the track quality based on the error covariance matrix of the Kalman filter for each confirmed track. For each confirmed track, the Kalman filtering 
process produces estimates of the location and velocity along with respective error covariances. As more correct associations are made to a track, the error covariance decreases and we can consider the track to be better estimated. The idea is that when a track is being estimated within some margin, the pinging can be partially focused on other areas of the search space or other tracks.

The basic flow of the algorithm is now described. If there are no confirmed targets, ping sequencing is determined by a sequential search pattern. When a confirmed track is formed from the MTT, we then evaluate the covariance matrix and compare the values for the track to a set threshold. If the error covariance for the velocity and location are less than their respective thresholds, we consider the track to be good quality and is not considered for the ping sequencing. If the track does not meet the thresholds then we select the source that gives the maximum signal excess for the predicted location of the track. For multiple confirmed tracks, we chose randomly with equal probability from the set of sources corresponding to each confirmed track.

Figure 4 shows tracking results without using ping sequencing. The ping sequence is a randomly chosen sequential pattern. The plot displays all the confirmed tracks (dark solid lines) throughout the length of the simulation. The false alarm rate is thirty for this case, which means that there are thirty false contacts per ping. The tracking algorithm was able to find a few segmented true tracks. However, during most of the duration of the simulation the targets were not tracked, and some false tracks were generated. In comparison, Fig. 5, clearly shows the benefits of intelligent ping sequencing. The target on the right was tracked for almost the entire length of the simulation, and although some false tracks were still confirmed, they were rejected upon repeat interrogation.

\subsection{Bandwidth Diversity}

We found that in large distributed sensor fields, tracking multiple targets was difficult with only one source pinging at a time as seen in Fig. 5. Having a high false alarm rate made the problem even worse. With only one source pinging at a time, the number of contacts for a target is so spread out over time that the tracking algorithm is unable to maintain a track or even find confirmed tracks. One way to increase the capability of the field is to take advantage of bandwidth diversity, meaning that multiple sources ping simultaneously, each using a separate frequency band.

Figure 6 shows tracking results for a case when three transmitters can ping simultaneously. Both true tracks were maintained throughout the duration of the scenario, showing improved results from those shown in Fig. 5 .

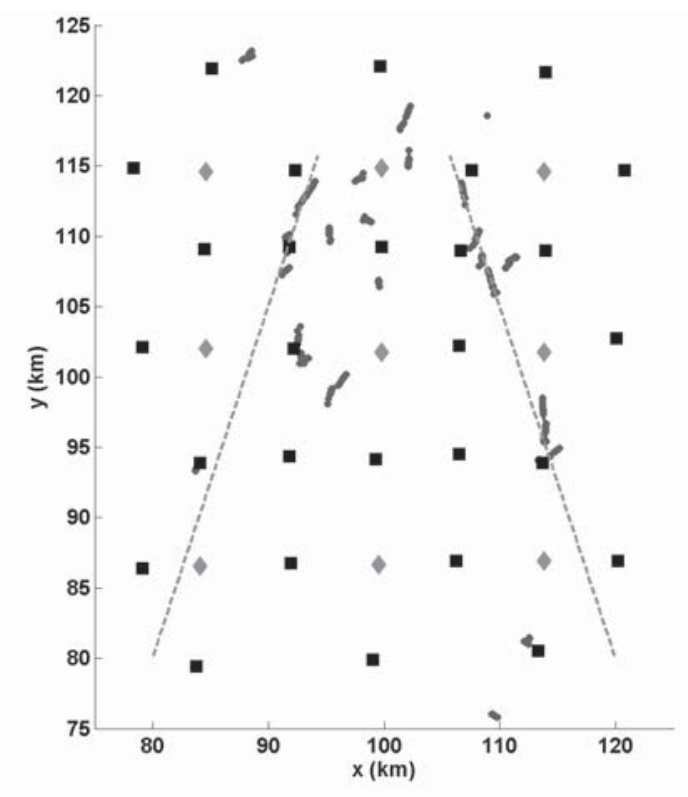

Figure 4: Tracking simulation results using a sequential pinging strategy. All confirmed tracks are displayed.

\section{Conclusion}

In this paper we proposed a metric to be used in the area search problem of a distributed sensor field and discussed ping sequencing strategies when targets are detected. It is clear from the results shown that intelligent ping sequencing is important for large sensor fields. We have shown two techniques for intelligent ping sequencing; track quality and bandwidth diversity. Ping sequencing does not need to be limited to these techniques and in future work we intend to explore other possibilities such as waveform diversity.

We seek to combine the probabilistic framework of $P_{T}$ with the heuristic approach of track quality to provide a balance between area search and tracking. We also plan to incorporate more realistic models in our simulations such as non-uniform target strength vs. aspect, depth dependence (sonobuoys and targets), and performance prediction uncertainty. In future research we will compare the probability of target presence metric to Monte Carlo simulations. We will also show more detailed tracking comparisons with benchmark tracking metrics to show the benefits of intelligent ping sequencing.

\section{Acknowledgments}

This work was funded by the U.S. Office of Naval Research, Contract Number N00014-01-G-0460, Delivery Order \#36.

\section{References}

[1] D. L. Hall. Mathematical Techniques in Multisensor Data Fusion. Artech House, Norwood, MA, 


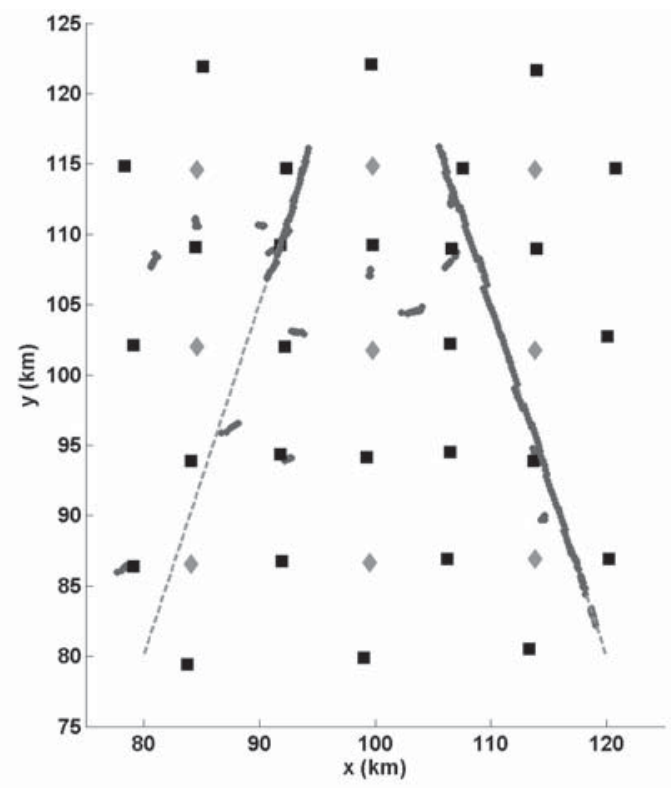

Figure 5: Tracking simulation results using an intelligent ping sequence. All confirmed tracks are displayed.

1992.

[2] P. L. Bogler. Radar Principles with Applications to Tracking Systems. John Wiley \& Sons, New York, 1990.

[3] F. Daum. Multitarget-Multisensor Tracking: Applications and Advances. Artech House, Norwood, MA, 1992.

[4] S. S. Blackman and R. Popoli. Design and Analysis of Modern Tracking Systems. Artech House, Norwood, MA, 1999.

[5] P. N. Ngatchou, W. L. J. Fox, and M. A. ElSharkawi. Distributed sensor placement with sequential particle swarm optimization. In Proc. IEEE Swarm Intelligence Symposiom 2005, pages 385-88, June 2005.

[6] R. J. Urick. Principles of Underwater Sound. McGraw Hill, New York, third edition, 1983.

[7] W. J. Albersheim. Close-form approximation to Robertson's detection characteristics. Proc. IEEE, 4473(7):839, 1981.

[8] S. Kanchanavally, R. Ordenoez, and J. Layne. Mobile target tracking by networked uninhabited autonomous vehicles via hospitability maps. In Proc. 2004 American Control Conf., pages 5570-75, June 2004.

[9] S. S. Blackman. Multiple Target Tracking with Radar Applications. Artech House, Norwood, MA, 1986.

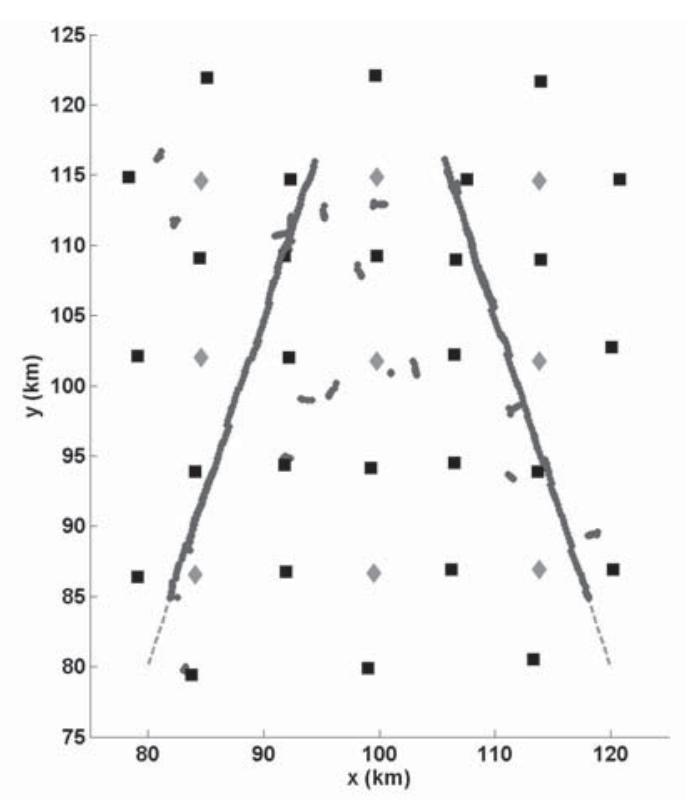

Figure 6: Tracking simulation results using bandwidth diversity and intelligent ping sequencing. All confirmed tracks are displayed. 